\title{
Injectable "nano-micron" combined gene-hydrogel microspheres for local treatment of osteoarthritis
}

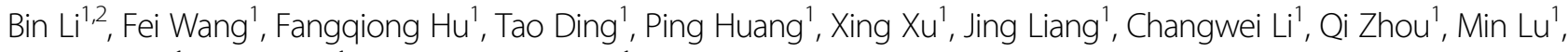
Lianfu Deng', Lei Guo (iD ${ }^{1}$ and Wenguo Cui ${ }^{1}$

\begin{abstract}
Sustained and controllable local gene therapy is a potential method for treating osteoarthritis (OA) through the delivery of therapeutic microRNAs (miRNAs) to targeted cells. However, direct injection of crude miRNAs for local gene therapy is limited due to its inadequate transfection efficiency, easy inactivation, and short half-life. Here, a multifunctional gene vector, arginine, histidine, and phenylalanine-modified generation 5 polyamidoamine (named G5-AHP), was employed to form G5-AHP/miR-140 nanoparticles by forming a complex with microRNA-140 (miR-140). Then, the nanoparticles were entrapped in hydrogel microspheres (MSs) to construct a "nano-micron" combined gene hydrogel to alleviate the degradation of articular cartilage. Monodisperse gelatin methacryloyl hydrogel MSs were produced under ultraviolet light using one-step innovative microfluidic technology. Evenly dispersed MSs showed better injectability in sustainable and matrix metalloproteinases (MMPs)-responsive degradation methods for local gene delivery. The G5-AHP/miR-140 nanoparticles released from the MSs exhibited high gene transfection efficacy and long-term bioactivity, facilitated endocytosis, and thus maintained the metabolic balance of cartilage matrix by promoting the expression of type II collagen and inhibiting the expression of a disintegrin and metalloproteinase with thrombospondin motifs-5 and MMP13 in chondrocytes. After injection of the "nano-micron" combined gene hydrogel into the articular cavity of the OA model, the gene hydrogel increased G5-AHP/miR-140 nanoparticle retention, prevented articular cartilage degeneration, and reduced osteophyte formation in a surgically induced mouse model of OA. The present study provides a novel cell-free approach to alleviate the progression of OA that shows potential for locally injected gene delivery systems.
\end{abstract}

\section{Introduction}

Gene therapy is a promising strategy for the treatment of human diseases and disorders (such as cancer and hereditary, musculoskeletal, and chronic or degenerative diseases) by delivering nucleic acids (DNA or RNA) to target tissues or cells ${ }^{1}$. However, the mode of delivering genes, which are crucial for successful gene therapy, is

\footnotetext{
Correspondence: Lei Guo (guolei607@126.com) or

Wenguo Cui (wgcui80@hotmail.com)

'Department of Orthopaedics, Shanghai Key Laboratory for Prevention and Treatment of Bone and Joint Diseases, Shanghai Institute of Traumatology and Orthopaedics, Ruijin Hospital, Shanghai Jiao Tong University School of Medicine, 197 Ruijin 2nd Road, Shanghai 200025, P. R. China

${ }^{2}$ Department of Orthopaedic Surgery, Changzheng Hospital, Second Military Medical University, Shanghai, China

These authors contributed equally: Bin Li, Fei Wang
}

often ignored ${ }^{2}$. Intravenous and oral administration is convenient and noninvasive and has been widely used in gene therapies. However, systemic delivery has a substantial risk of off-target effects and requires a higher dose to sustain the therapeutic effect, resulting in limited application of systemic delivery in tissue-specific diseases $^{3}$. Therefore, the gene therapy field has recently entered a new technological era in which multiple local delivery approaches have been applied to improve the distribution of therapeutic genes in target tissues ${ }^{2,4-6}$.

The local gene delivery method has been applied in numerous studies to improve the efficacy of gene therapy vectors by providing high regional concentrations of genes, enhancing transfection efficiency, decreasing potential side effects, and circumventing substantial 
systemic delivery barriers ${ }^{7}$. Numerous opportunities also exist to develop novel local delivery systems that can release genes sustainably and controllably due to their intrinsic shortcomings, such as the limited half-life of genes, aberrant distributions and invasiveness of frequent injections ${ }^{8,9}$. Thus, appropriate biomaterials are essential to establish stable and regulatable gene expression in a spatiotemporally precise manner ${ }^{10,11}$. Hydrogels act as key candidates to form a hybrid biomaterial system due to their superior biocompatibility and similarity to biological tissues $^{12,13}$. However, bulk hydrogels for injectable delivery applications need special approaches, including in situ and shear thinning crosslinking, to avoid possible leakage before gel formation and delivery failures due to fast gelation ${ }^{14}$. Hydrogel microspheres (MSs) are attractive in minimally invasive biomedical applications and are injected with small needles because of their small size. Microfluidics exhibits excellent advantages over batch emerging, mechanical fragmentation and lithography approaches to form monodisperse hydrogel MSs by emulsifying an aqueous hydrogel phase within a paraffin oil phase at the junction of microchannels in designed configurations $^{15,16}$. Microfluidic hydrogel MSs with the advantage of excellent injectability without surplus procedures are extremely compatible with local gene therapy, such as percutaneous manipulation and intrapleural and intra-articular injection ${ }^{17,18}$.

Osteoarthritis $(\mathrm{OA})$ is the most prevalent degenerative joint disease and a major cause of pain and disability in adults $^{19}$. Destruction of articular cartilage is a characteristic feature of OA, which is caused by increased production of matrix metalloproteinases (MMPs) and a disintegrin and metalloproteinase with thrombospondin motifs (ADAMTS) ${ }^{20,21}$. Recently, gene therapy delivering microRNAs (miRNAs) has been a novel therapeutic method in the treatment of joint degenerative diseases $^{22,23}$. MicroRNA-140 (miR-140) is specifically expressed in cartilage and plays a critical role in chondrocyte development and homeostasis ${ }^{24-26}$. Therefore, gene therapy delivering miR-140 into cartilage can delay cartilage degeneration and alleviate the disease. OA mainly affects weight-bearing joints and has minimal extra-articular symptoms. Therefore, local intra-articular injection is an attractive treatment approach for $\mathrm{OA}^{27,28}$. Under UV irradiation, the prepared homogeneous gelatin methacryloyl (GelMA) MSs photocrosslinked with its integral size kept at the micron level and its internal porous structure sustained at the nano level, similar to bulk hydrogels. MiR-140 can be encapsulated within MSs, thus establishing a localized sustainable and controllable gene delivery system by responding to increasing MMPs in OA joint capsules ${ }^{29-32}$. However, poor transfection efficacy and the rapid degradation of nucleic acids in vivo exist in the therapeutic applications of naked miR-140 gene-hydrogel MSs. Thus, it is necessary to design an efficient and stable gene delivery vector that can prolong the existence time and provide high transfection efficacy of the functional miRNAs in the target tissue ${ }^{33}$.

Cationic dendrimers are a nonviral vector for gene delivery with low immunogenicity and excellent penetration $^{34,35}$. Moreover, amino acid conjugation is a common strategy to enhance the gene transfection efficacy of cationic polymers ${ }^{36}$. Dendrimers modified with Arg, Phe, and His show a synergistic effect on gene delivery. Arg can improve polyplex formation and stability, Phe can increase the cellular internalization of polyplexes by modulating the balance of charge and hydrophobic contents on dendrimers, and His facilitates the endosomal escape of polyplexes ${ }^{37}$. G5-AHP was designed by facile chemistry to transfer miR-140 into target cells, which could facilitate endocytosis and improve transfection efficiency. Furthermore, G5-AHP protected miR-140 from nuclease cleavage and enhanced its stability in target cells.

In this study, injectable "nano-micron" combined genehydrogel MSs were constructed by imbibing G5-AHP/ miR-140 nanoparticles into the microfluidic MS local delivery system to alleviate OA progression (Fig. 1). First, the high efficacy and stable G5-AHP/miR-140 nanoparticles improved the miR-140 transfer efficiency and maintained its biological activity for a long time. Second, the local intra-articular injection of gene-hydrogel MSs could minimize the possibility of off-target effects and reduce the amount of gene drug delivery. Third, the "nano-micron" combined GelMA MS delivery system sustained the long-acting release of G5-AHP/miR-140 nanoparticles in an MMP-responsive manner. The degradation of GelMA MSs consumed the preexisting MMPs in the OA joint capsule. The G5-AHP/miR-140 nanoparticles increased the expression of COL2 and decreased the expression of MMPs and ADAMTS in osteoarthritic chondrocytes, which alleviated the degradation of the cartilage matrix. Finally, intra-articular injection of "nano-micron" combined gene-hydrogel MSs obviously alleviated the progression of OA in mice. Taken together, these results indicate that the injectable "nanomicron" combined gene-hydrogel MSs have good application potential for the treatment of OA.

\section{Results and discussion}

\section{The multifunctional efficient and protective cationic dendrimer G5-AHP}

Multifunctional gene vectors of G5-AHP were synthesized by a one-pot facile condensation reaction and were employed here for miR-140 loading and transfection (Fig. 1a). According to ${ }^{1} \mathrm{H}$ NMR spectrum analysis of G5-AHP (Fig. 2a), three different amino acids were successfully conjugated on the dendrimer surface, and the 
a
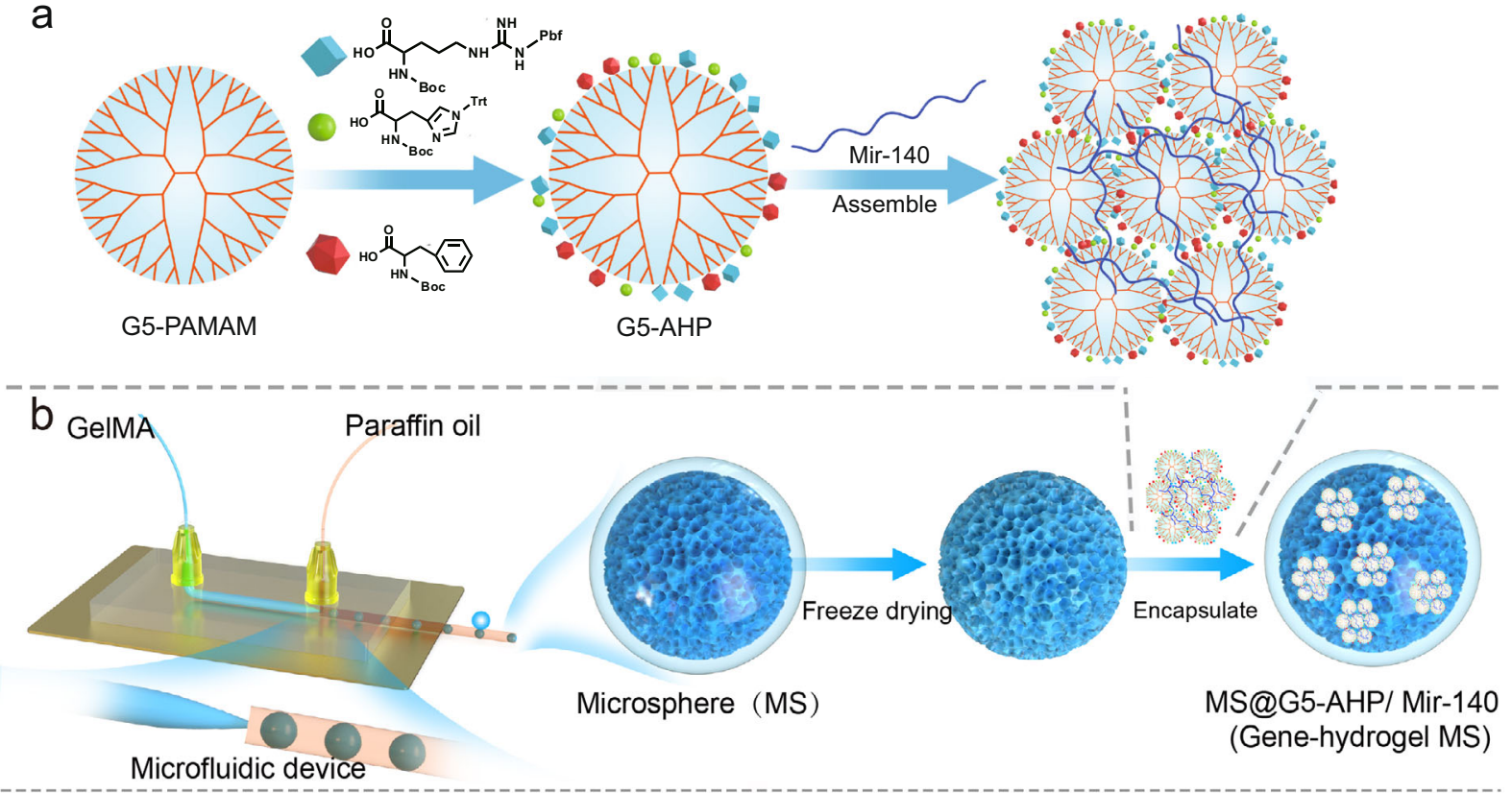

C
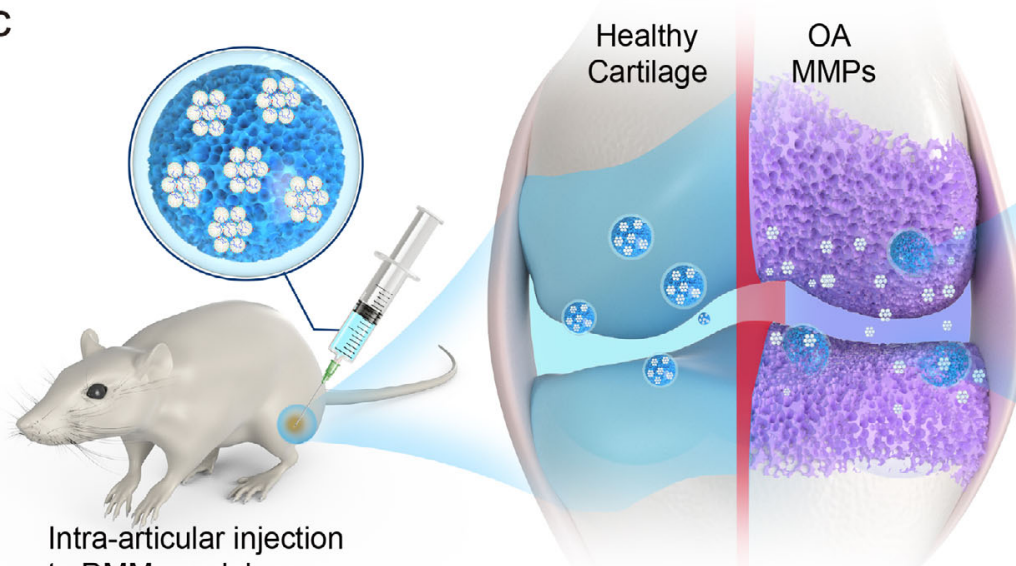

to DMM model mouse

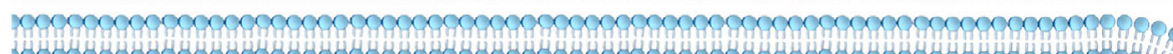

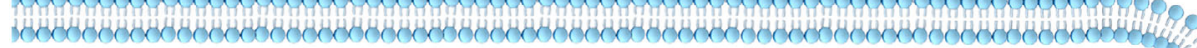

d
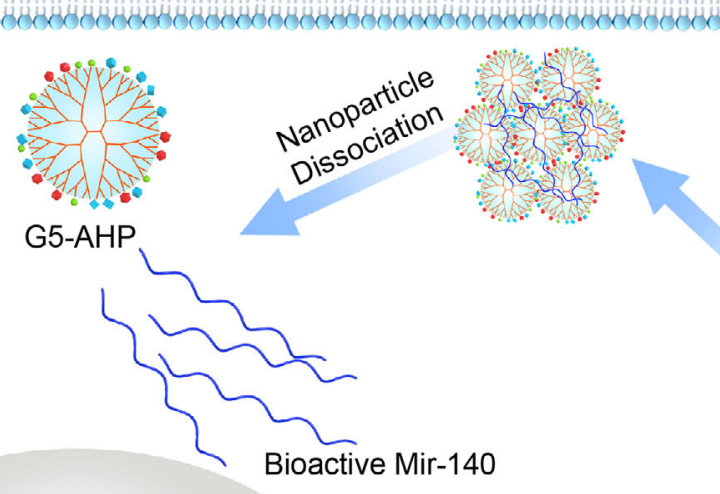

Nucleus

Bioactive Mir-140

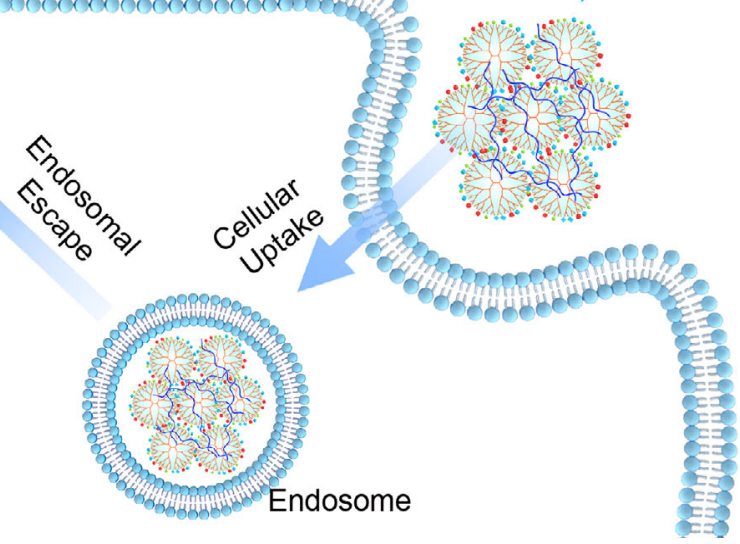

Fig. 1 Gene-hydrogel microspheres for the treatment of OA. a Synthesis of G5-AHP and G5-AHP/miR-140. b Gene-hydrogel MSs. c MS@G5-AHP/miR140 was injected into the articular space to alleviate the progression of OA. $\mathbf{d}$ Endocytosis of G5-AHP/miR-140 polyplexes and the release of miR-140. 


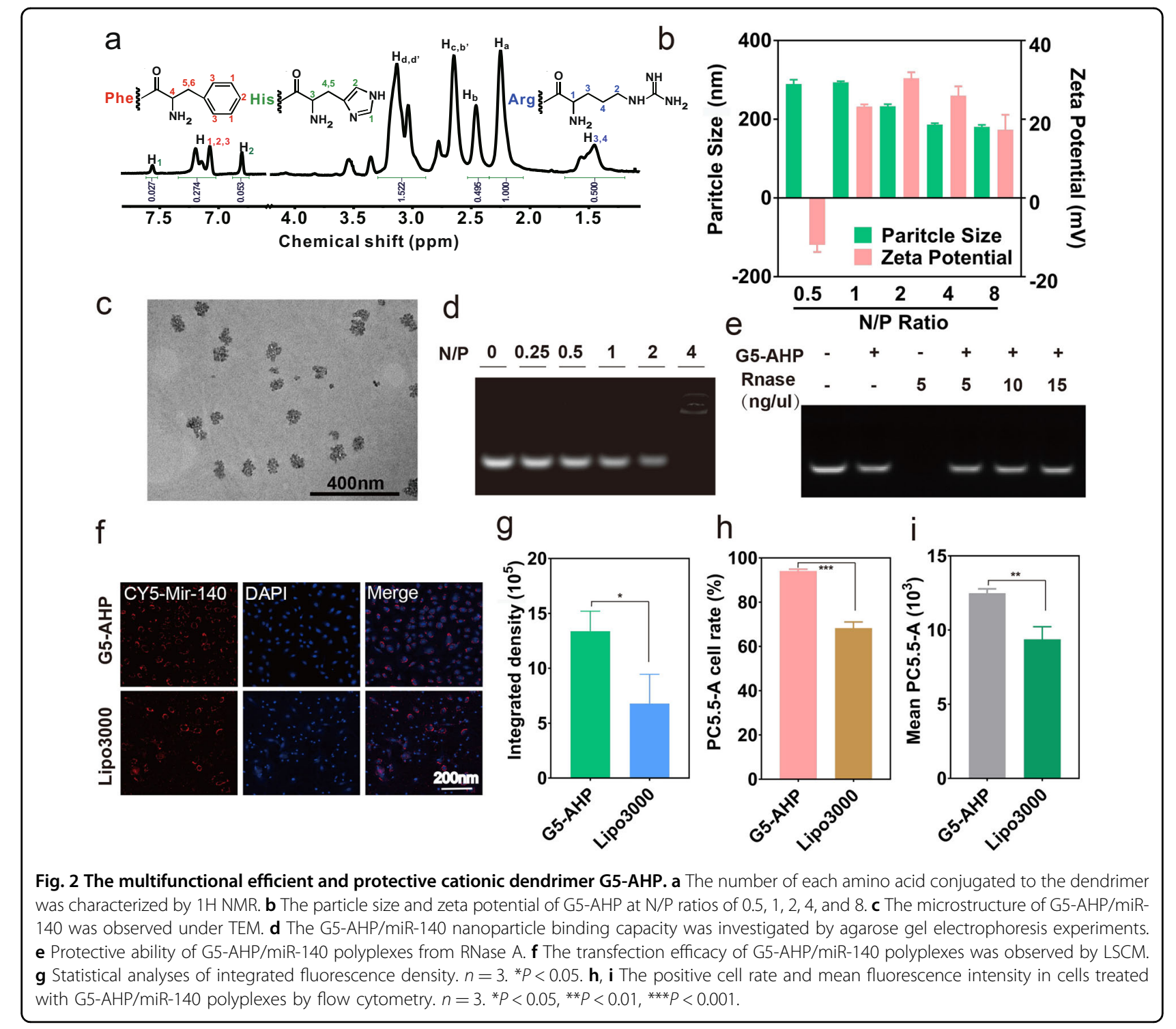

number of each amino acid was calculated by integration of the dendrimer and amino acid proton spectra. The average numbers of arginine-, histidine-, and phenylalanineconjugated dendrimers were 59,25 , and 16 , respectively ${ }^{38}$. G5-AHP/miR-140 polyplexes were prepared using an assembly strategy by mixing. As shown in Fig. 2b, the hydrodynamic diameter of the complexes gradually decreased to approximately $200 \mathrm{~nm}$ as the $\mathrm{N} / \mathrm{P}$ ratio ranged from $0.5: 1$ to $8: 1$, and the charge state of the complexes became positive at an N/P ratio of 1:1. The microstructures of G5, G5-AHP, and G5-AHP/miR-140 were also observed under a transmission electron microscope (TEM) (Fig. 2c and Supplementary Fig. S1), and the results revealed that the size of the formed G5-AHP/miR-140 nanoparticles increased obviously compared to that of G5-AHP. These properties of G5-AHP/miR-140 polyplexes are essential for cellular uptake and highly efficient gene transfection ${ }^{39}$. The size of the G5-AHP/miR-140 nanoparticles was almost unaltered under gentle stirring for 4 days, indicating that miR-140 nanoparticles exhibit good stability (Supplementary Fig. S2). Agarose gel electrophoresis experiments were used to evaluate the binding characteristics and protective capacity of G5-AHP to miR-140. As shown in Fig. 2d, the polyplexes obviously reduced miR-140 mobility at an N/P ratio of more than 2:1. Moreover, these structures protected miR-140 from digestion by RNase A at concentrations of 5,10 , and $15 \mathrm{ng} / \mathrm{ml}$ (Fig. 2e). In addition, protonable tertiary amine groups in the dendrimer interior at high density can provide the "proton sponge effect" during the endosomal escape process ${ }^{40}$. The gene transfection efficacy of G5-AHP was measured by laser scanning confocal microscopy (LSCM) and flow cytometry tests. 
The gene transfection efficiency and cytotoxicity of G5-AHP/miR-140 increased with the increasing N/P ratio (Supplementary Fig. S3). In consideration of the efficiency and cytotoxicity, an N/P ratio of 4 was selected as the optimal condition for further study. Compared with commercialized Lipofectamine 3000, the G5-AHP/miR-140 polyplexes showed excellent cellular uptake and transfection efficiency. The fluorescence intensity of transfected cells by G5-AHP/miR-140 was superior to that of Lipofectamine 3000 (Fig. 2f, g). Flow cytometry revealed that the positive PC5.5-A cell rate (Cyanine5-labeled miR-140 cell rate) and mean fluorescence intensity in the cells transfected with G5-AHP/Cyanine5-labeled miR-140 (CY5-miR140) polyplexes were also obviously higher than those of the cells transfected with Lipofectamine 3000 (Fig. 2h, i). These results reveal that G5-AHP, with high gene transfection efficacy and low cytotoxicity, is an excellent gene delivery vector for gene therapy.

\section{Intracellular function of G5-AHP/miR-140 nanoparticles in vitro}

Articular cartilage is a special and avascular connective tissue that covers joint surfaces and consists of type II collagen and proteoglycans. Articular chondrocytes are the only cell type in cartilage and are responsible for the synthesis of cartilage extracellular matrix $(E C M)^{41}$. Previous studies indicated that excessive tensile strain $(>10 \%)$ had negative effects on chondrocyte ECM synthesis, which plays an important role in the development of $\mathrm{OA}^{42}$. Thus, protecting chondrocytes from tensile strain-induced imbalance and degradation of ECM synthesis is critical for treating OA. In this study, the cyclic tensile strain was loaded on chondrocytes to mimic cartilage loading in vivo. The effects of cyclic tensile strain on the metabolism of chondrocytes that were incubated in G5-AHP/miR-140 polyplexes were investigated. Real-time quantitative PCR analysis indicated that the expression of COL2 was significantly increased, and the expression of MMP13 and ADAMTS5 was obviously reduced in the chondrocytes treated with G5-AHP/miR-140 compared to the untreated cells $($ Fig. $3 \mathrm{a}-\mathrm{c})$. Similar results were obtained by western blot analysis and immunofluorescence assays (Fig. 3d-m). Taken together, these results suggest that G5-AHP/miR140 transfection could effectively maintain chondrocyte function in vitro under cyclic tensile strain conditions.

\section{Fabrication and characterization of gene-hydrogel microspheres for local delivery systems}

GelMA MSs were produced by a microfluidic device (Fig. 1b). The device is simple, practical, and widely used in many studies ${ }^{17}$. The GelMA solution was used as the dispersed phase, while paraffin oil was used as the continuous phase. The shear force exerted by the paraffin oil channel conducted a split-up of the GelMA solution, receiving mixed droplets. The receiving droplets were photocrosslinked into MSs under UV irradiation. The microfluidic method can produce highly uniform and size-controllable MSs compared with the traditional emulsification method. The diameter of the MSs can be adjusted by changing the flow rate ratios of the continuous channel $(\mathrm{Qc})$ and dispersed channel (Qd). Here, uniform GelMA MSs can be obtained when the flow rate ratio is 10:1 (Qd:Qc). After freeze-drying, anhydrous GelMA MSs can imbibe polyplex solutions to form G5AHP/miR-140-loaded MS (MS@G5-AHP/Mir-140) gene hydrogels to alleviate OA in vivo. Nearly all G5-AHP/ miR-140 nanoparticles were encapsulated into the MS hydrogel carrier as previously reported ${ }^{43,44}$. According to the morphological characterization observed by brightfield microscopy (BM), MSs, and MS@G5-AHP/miR-140 were almost spheroidal and uniform (Fig. 4a). The size of the MSs was approximately $150 \mu \mathrm{m}$, and the microstructures of the lyophilized MSs and MS@G5-AHP/miR140 were visualized using a scanning electron microscope (SEM) (Fig. 4b). We could directly observe G5-AHP/miR140 attached to MSs as nanoscale spots, as shown in Fig. 4b. To verify whether G5-AHP/miR-140 was absorbed into MSs, we synthesized fluorescein isothiocyanate (FITC)-conjugated GelMA MSs and CY5-miR-140. FITCMS@G5-AHP/CY5-miR-140 was colocalized using LSCM (Fig. 4c). When suspended in an aqueous solution, MS@G5-AHP/miR-140 dispersed and could be injected into the articular cavity by using an injector (Fig. $4 \mathrm{~d}$ ). To examine the application potential of the developed MS@G5-AHP/miR-140, we first investigated the cytotoxicity of MSs and MS@G5-AHP/miR-140 on primary mouse chondrocytes using the CCK-8 test and Live/Dead staining at 1, 3, and 5 days. As shown in Fig. 4e, the CCK8 test showed that there was no significant difference in cell viability and proliferation between the experimental group and the control group at different time points. In addition, the live/dead cell assay showed that the seeded cells were almost all alive, and only a few dead cells were observed at all time points (Fig. 4f). Furthermore, the cell density was increased from 1 to 5 days, as evidenced by a significant increase in the number of viable cells (Fig. 4g). These results suggest that MS@G5-AHP/miR-140 had excellent biocompatibility with chondrocytes.

\section{Sustainable and controllable release of gene-hydrogel microspheres}

GelMA MSs are an ideal vector for intra-articular injection due to their excellent biocompatibility and controllable size. The release rate of the therapeutic gene cargo inside GelMA MSs can be regulated by MMP activity at different phases of OA. Transwell chamber assays were used to verify the MMP-responsive release of GelMA MSs (Fig. 5a). MSs were incubated with $10 \mathrm{ng} / \mathrm{ml}$ 


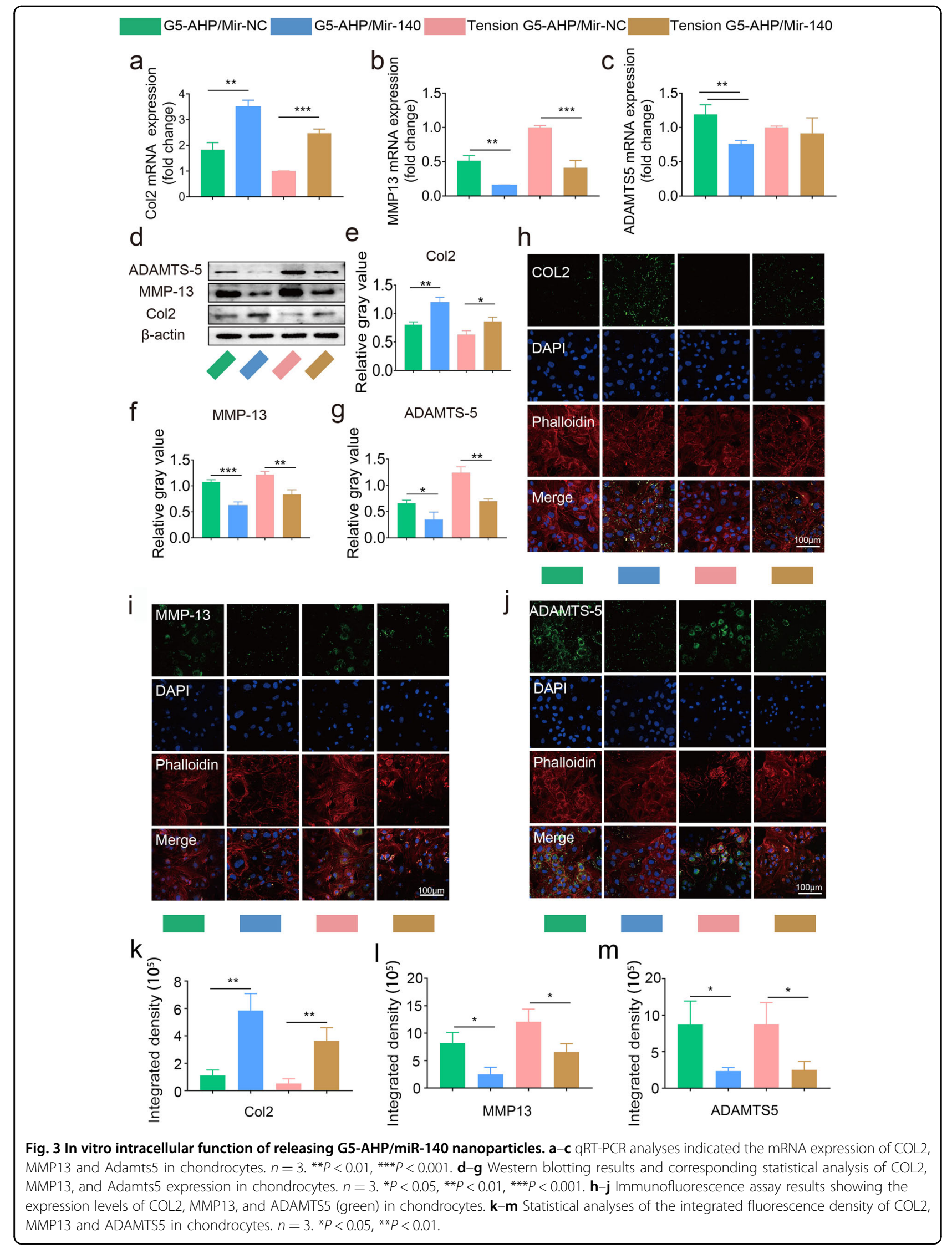




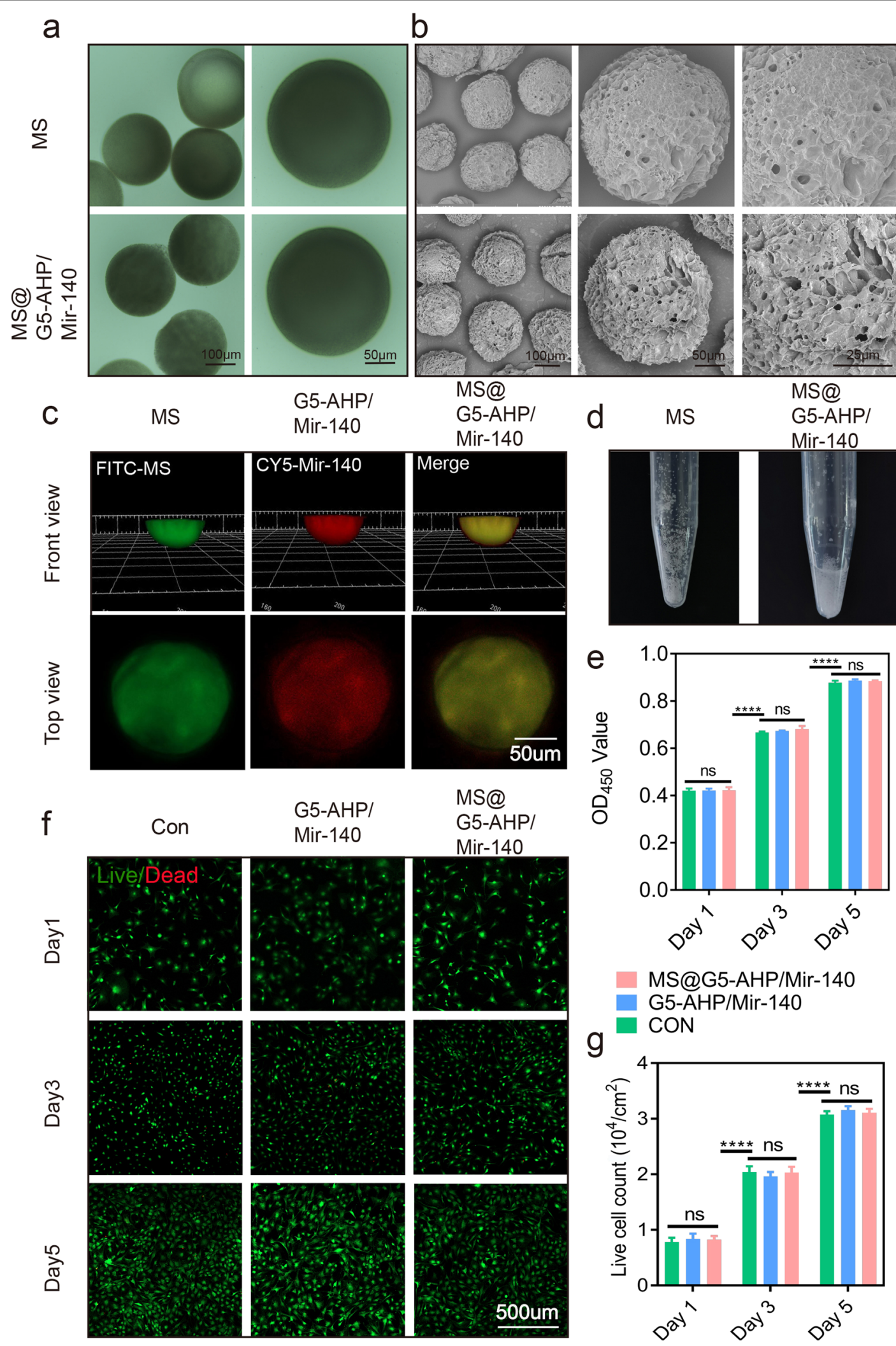

Fig. 4 (See legend on next page.) 
(see figure on previous page)

Fig. 4 Characterization of gene-hydrogel microspheres (MS@G5-AHP/miR-140) for local delivery systems. a The particle size and morphology of MSs and MS@G5-AHP/miR-140 in the swollen state measured by BM. b The microstructures of lyophilized MSs and MS@G5-AHP/miR-140 were observed under SEM.c FITC-MS@G5-AHP/Cy5-miR-140 was colocalized by LSCM.d Macroscopic view of GelMA MSs and MS@G5-AHP/miR-140.e The OD value of CCK-8 tests shows the cell viability and proliferation of chondrocytes treated with G5-AHP/miR-140 and MS@G5-AHP/miR-140. n=3. NS no significance. ${ }^{* * *} P<0.0001$. $\mathbf{f}$ Representative images showing the live/dead assay of chondrocytes cocultured with G5-AHP/miR-140 and MS@G5AHP/miR-140. g Quantitative analysis of live cells in live/dead assays. $n=3$. NS no significance. ${ }^{* * *} P<0.0001$.

MMP solution for $1,3,5$, and 7 days to simulate the progressive degradation process in vivo, which was observed by BM. According to its morphological changes, only a few changes were observed in the morphology of the MSs treated with MMPs on the first day, and the MSs were black. On the third day, some cracking emerged around the MSs, and the color became lighter. On the fifth day, the morphology of the MSs started to collapse with irregular margins, and the color became light. On the seventh day, the MSs almost disappeared because most of their substances were lost. MSs dispersed in phosphatebuffered saline (PBS) without MMPs remained black and nearly intact with few cracks at day 7 (Fig. 5b). The degradation process of the MSs was also observed by SEM, and the results coincide with the processes observed by BM (Supplementary Fig. S4). LSCM showed the endocytosis of CY5-miR-140 in chondrocytes cocultured with MS@G5-AHP/miR-140 treated with and without MMPs for 2 days. The group treated with MMPs engulfed more CY5-miR-140 than the untreated group (Fig. 5c). This result indicates that MS@G5-AHP/miR-140 responds to MMPs and ensures that G5-AHP/miR-140 released from MS@G5-AHP/miR-140 is bioactive. The degradation process and drug release kinetics were investigated to evaluate the applicability of MS@G5-AHP/ miR-140 as a drug delivery vehicle and advance MMP responsiveness. As illustrated in Fig. 5d, the release profiles revealed that MS@G5-AHP/miR-140 treated with MMPs showed a quick release phenomenon and was almost entirely released on the fifth day. In contrast, MS@G5-AHP/miR-140 without MMPs showed longterm release performance and sustainable release for more than 2 weeks. Collectively, these results demonstrate that MS@G5-AHP/miR-140 exhibits MMP-responsive characteristics and constant release within the degradation period, providing a superb approach for intraarticular injection for OA treatment.

\section{In vivo therapeutic effect of osteoarthritis}

Destabilized medial meniscus (DMM) surgery has been widely used to establish animal models of OA. One week after DMM surgery, the mice were injected with PBS, MS@G5-AHP/miR-NC, G5-AHP/miR-140, and MS@G5AHP/miR-140 once every 2 weeks. The mice were sacrificed at 12 weeks post surgery. X-ray radiographs of the mouse joints were acquired at 1 and 12 weeks after DMM surgery. No signs of acute infectious inflammation were observed in any group. Joint space widths of knee radiographs were measured. The results showed that the medial joint space between the femur and tibia was normal at 1 week (Fig. 6a, b). The joint space narrowed at 12 weeks after DMM surgery in the five groups, whereas the MS@G5-AHP/miR-140 group had a wider articular space than the PBS and MS@G5-AHP/miR-NC groups (Fig. 6a, c). The X-ray images showed a favorable change in the therapeutic effect in the MS@G5-AHP/miR-140 group. The reconstructed micro-CT results directly revealed that osteophyte formation, indicated with red circles, was obviously reduced in the MS@G5-AHP/miR-140 group compared to the PBS group, MS@G5-AHP/miR-NC group, and G5-AHP/miR-140 group (Fig. 6d). The radiography results indicated that MS@G5-AHP/miR-140 had a better therapeutic effect on DMM-induced OA.

To further investigate the therapeutic effect of MS@G5-AHP/miR-140 on OA, we performed histological assessments of cartilage tissues by hematoxylineosin staining, toluidine blue staining, and safranin Ofast green staining (Fig. 7a-c). Typical structural features of OA, including surface discontinuity, denudation erosion, and deformities, were observed in the PBS group and G5-AHP/miR-NC group compared with the PBS group. Specifically, the MS@G5-AHP/miR-140 group showed the greatest morphological integrity with less severe lesions, decreased surface denudation, and increased tissue cellularity and cloning. Based on the results of toluidine blue staining and safranin Ofast green staining, glycosaminoglycan (GAS) in the five groups was analyzed (Fig. 7d). The MS@G5-AHP/miR140 group had the best outcome with regard to the GAS level, indicating good retention of cartilage thickness. In addition, the G5-AHP/miR-140 group and MS@G5AHP/miR-140 group had lower OARSI scores than the PBS group (Fig. 7e) and showed better results with approximately $14.6 \%$ and $42.2 \%$ reductions. In addition, the depth of cartilage macroscopic lesions was reduced in the G5-AHP/miR-140 group and MS@G5-AHP/miR140 group by approximately $29.2 \%$ and $48.2 \%$, respectively, compared with the PBS group (Fig. 7f). Furthermore, the expression levels of COL2 and MMP13, two typical markers of articular cartilage, were evaluated by 

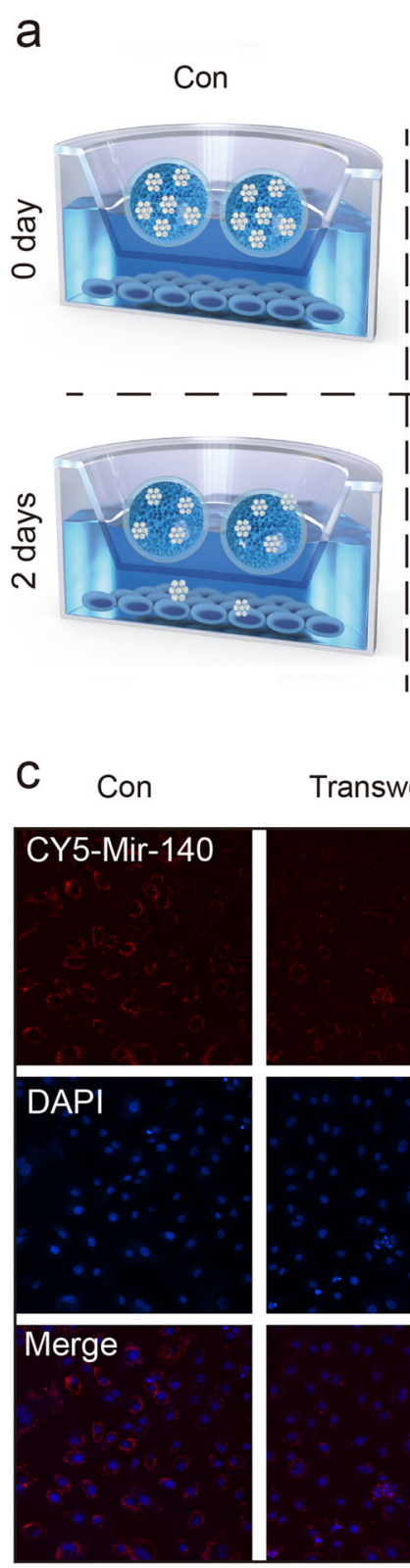

Transwell
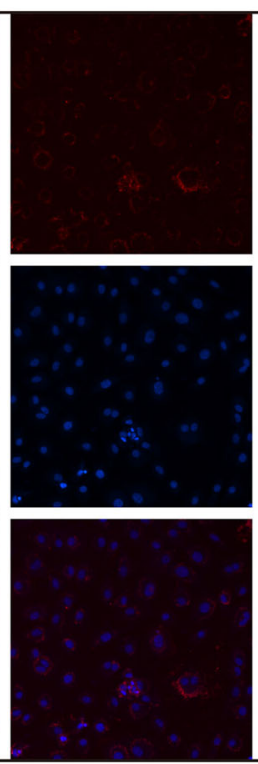

Transwell +MMPs
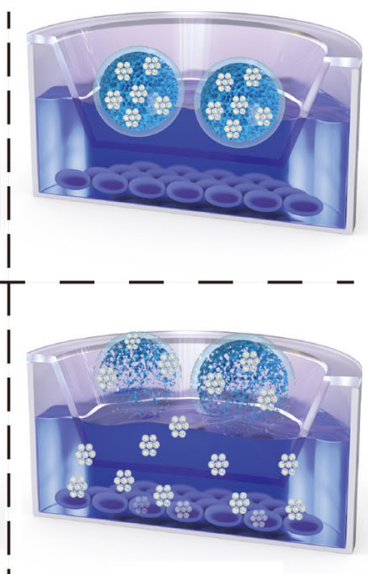

Transwell + MMPs
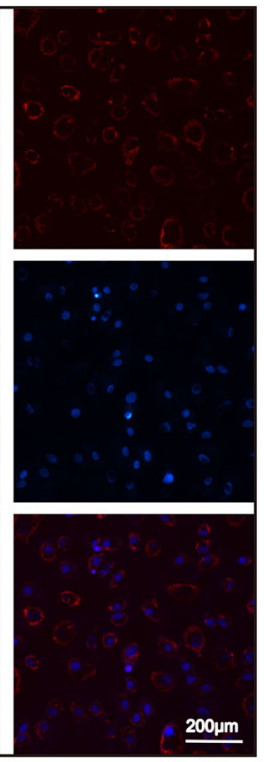

b

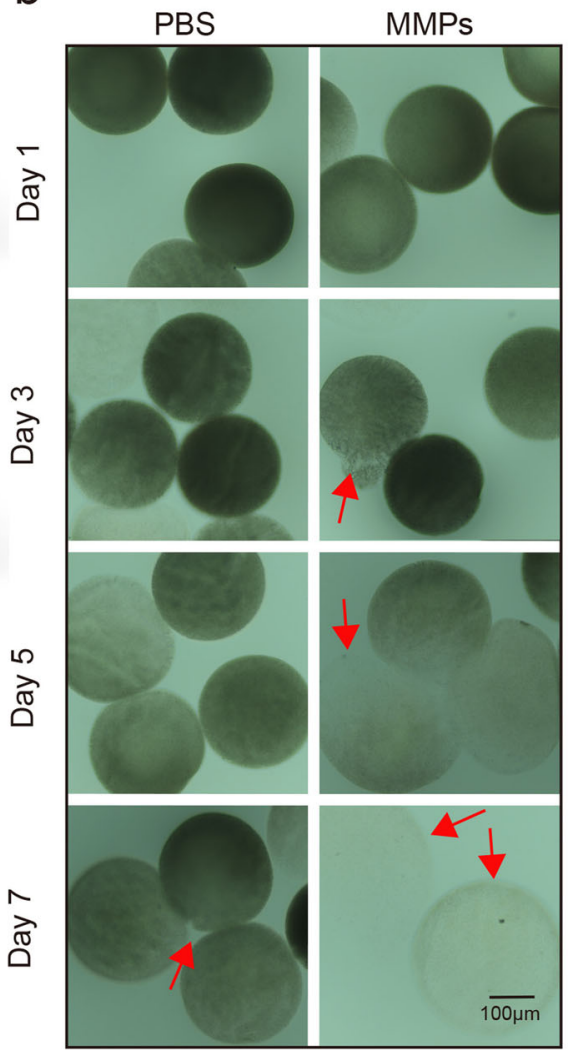

d

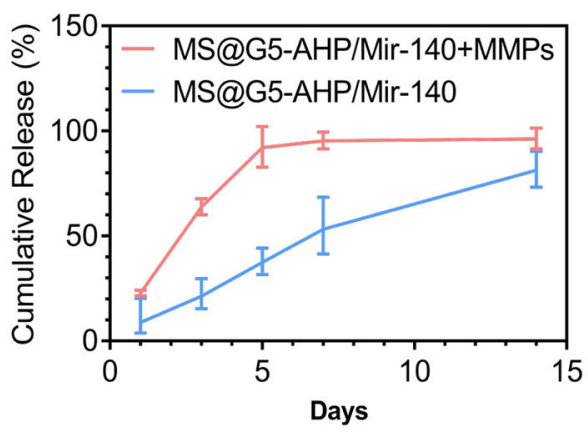

Fig. 5 Sustainable and MMP-responsive release of MS@G5-AHP/miR-140. a The degradation and responsive release process of MS@G5-AHP/ miR-140 microgels with or without MMPs using Transwell chambers. b Morphological changes in the microspheres after treatment with MMPs. c The transfection effect of G5-AHP/miR-140 after release from MS@G5-AHP/miR-140 microgels treated with or without MMPs. d The cumulative release curve of MS@G5-AHP/miR-140.

immunohistochemical assays. The expression of COL2 was decreased in the PBS group and MS@G5-AHP/miR$\mathrm{NC}$ group, and this change was reversed by G5-AHP/ miR-140. The expression of COL2 was the highest in the MS@G5-AHP/miR-140 group compared with the other groups. The opposite results were observed in the measurement of the expression of MMP13 (Supplementary Fig. S5). All these results indicate that MS@G5AHP/miR-140 can delay the degeneration of articular cartilage and inhibit the process of $\mathrm{OA}$ in the DMM model of OA.

\section{Conclusions}

In this study, G5-AHP/miR-140 nanoparticles were innovatively fabricated to alleviate the progression of OA by downregulating the expression of MMP13 and ADAMTS5 in chondrocytes. Moreover, GelMA MS hydrogel synthesized by one-step microfluidics 


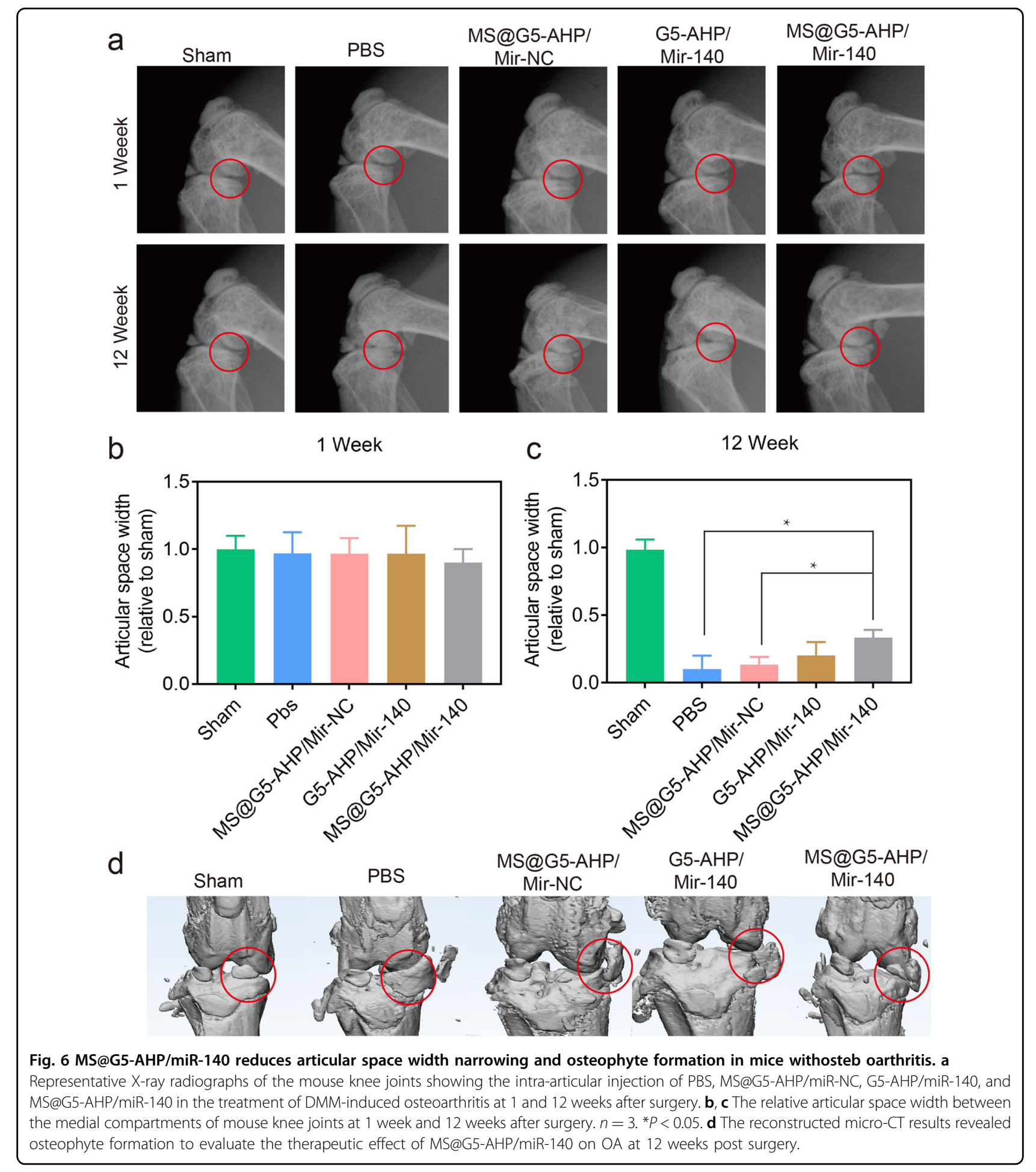

technology was used to deliver gene nanoparticles in a sustainable and controllable manner for local gene therapy. The present study demonstrated that MS@G5AHP/miR-140 gene-hydrogel MSs with excellent biocompatibility could protect chondrocytes from mechanical tension-induced degeneration and delay the development of OA. Furthermore, injectable responsive MS@G5-AHP/miR-140 "nano-micron" combined microfluidic gene-hydrogel MSs, as a novel regional gene therapy strategy, may be effective in treating localized diseases such as fractures, tenosynovitis, and cataracts. 


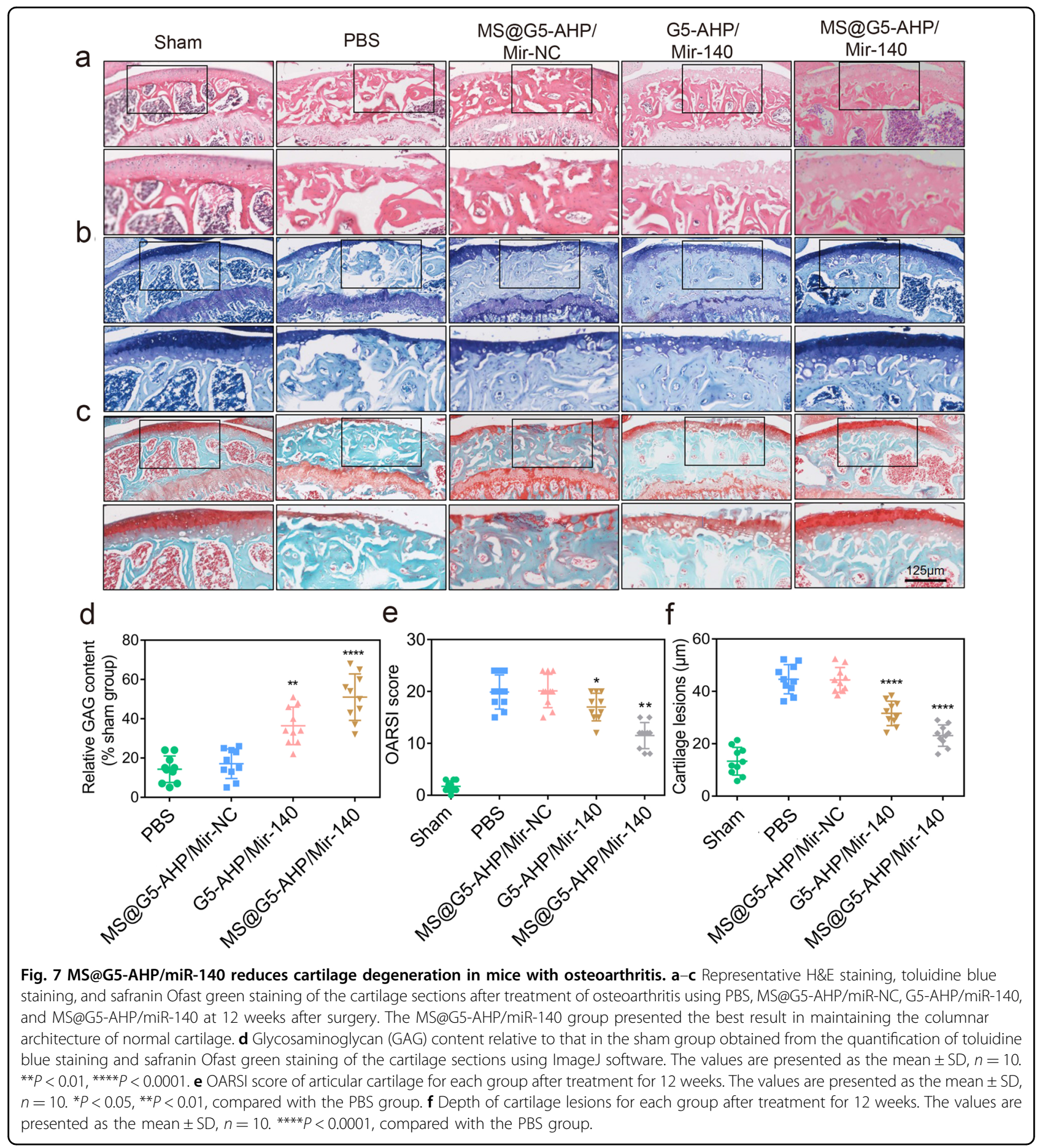

\section{Materials and methods}

\section{Materials and reagents}

Gelatin from porcine skin (type A) was purchased from Sigma-Aldrich (St. Louis, MO) and used as received. Amine-terminated G5 PAMAM dendrimers were purchased from Dendritech (Midland, MI). Sodium periodate, dicyclohexylcarbodiimide, and $\mathrm{N}$-hydroxysuccinimide were purchased from Macklin
Reagent Co. (Shanghai, China). Adipic dihydrazide and Lipofectamine 3000 were obtained from Invitrogen (Carlsbad, California). MiR-140 and Cyanine5 (CY5)miR-140 were designed and synthesized by GenePharma (Shanghai, China). RNase A and RNase inhibitor were purchased from Beyotime Biotechnology (Shanghai, China). All other chemicals were used as received without further purification. 


\section{Preparation and characterization of the G5-AHP/miR-140 complexes}

G5-AHP dendrimers were synthesized as previously reported $^{37}$. The purified product of G5-AHP was freezedried and characterized by ${ }^{1} \mathrm{H}$ NMR in $\mathrm{D}_{2} \mathrm{O}$ (Avance III, Bruker $600 \mathrm{MHz}$, Germany). The complexes of G5-AHP/ miR-140 were prepared by mixing G5-AHP and miR-140 at different $\mathrm{N} / \mathrm{P}$ ratios. The $\mathrm{N} / \mathrm{P}$ ratio was calculated according to the cationic groups (N number) on the G5AHP surface to anionic phosphate groups ( $\mathrm{P}$ number) of miR-140. All the G5-AHP/miR-140 complexes were incubated for $10 \mathrm{~min}$ at room temperature followed by characterization and activity evaluation. The morphology of the G5-AHP/miR-140 complex was observed by TEM (Hitachi, HT7700), and their particle size and zeta potential at N/P ratios of $0.5: 1,1: 1,2: 1,4: 1$, and 8:1 were determined on a Malvern Zetasizer Nano ZS90 (Malvern, UK) at $25^{\circ} \mathrm{C}$. The binding capacity of miR-140 and the G5-AHP dendrimer was estimated by agarose gel (Biowest, Spain) electrophoresis. G5-AHP/miR-140 complexes (N/P ratios of $0: 1,0.25: 1,0.5: 1,1: 1,2: 1$, and $4: 1$ ) were electrophoresed on a $2 \%(\mathrm{w} / \mathrm{v})$ agarose gel at $100 \mathrm{~V}$ for $20 \mathrm{~min}$. The miR-140 in the gels was stained with ethidium bromide and visualized under UV illumination (Tanon-2500, China). For the RNase A protection assay, G5-AHP/miR-140 complexes at an N/P ratio of 1:1 were incubated with RNase A at final concentrations of 5, 10, and $15 \mathrm{ng} / \mu \mathrm{l}$. Afterwards, RNase inhibitors were used to eliminate the effect of RNase A. Then, the solutions were further analyzed using agarose gel electrophoresis as described above.

\section{Fabrication of hydrogel MS and MS@G5-AHP/miR-140}

Methacrylic-modified gelatin (GelMA) was synthesized as previously reported ${ }^{45}$. In brief, methacrylic acid was added dropwise to the gelatin solution (dissolved in PBS), and the reaction solution was stirred for $1 \mathrm{~h}$ at $50{ }^{\circ} \mathrm{C}$. After that, $5 \times$ dilution PBS was added, and the mixture was extensively dialyzed against distilled water for 1 week at $40{ }^{\circ} \mathrm{C}$. The purified GelMA porous foam was prepared by the freeze-drying method and stored at $-80^{\circ} \mathrm{C}$. GelMA MSs were prepared using a modified microfluidic flowfocusing device ${ }^{46,47}$. Briefly, GelMA (5 wt\%) and photoinitiator $(0.5 \mathrm{wt} \%)$ were dissolved in PBS as the aqueous phase, and paraffin oil containing $5 \mathrm{wt} \%$ Span 80 was used as the oil phase. They were injected into inlets of the microfluidic device from syringes. The flow rates of both the aqueous phase and oil phase were controlled by a syringe pump (Lead Fluid, China). The generated emulsion droplets were irradiated for $10 \mathrm{~min}$ by UV light $(254 \mathrm{~nm})$. The MSs were collected into $50 \mathrm{ml}$ centrifuge tubes, suspended for $10 \mathrm{~min}$ to remove the paraffin oil as much as possible, and then washed with acetone and PBS three to five times to remove the paraffin oil and other additives. The GelMA MSs were then freeze-dried to absorb G5-AHP/miR-140 solutions, forming the MS@G5AHP/miR-140 microgels.

\section{Physical characterization test of MS@G5-AHP/miR-140}

(I) BM: the particle size and morphology of MSs and MS@G5-AHP/miR-140 in the swollen state were measured by BM (LSM800, Zeiss, Germany).

(II) SEM: MSs and MS@G5-AHP/miR-140 were frozen at $-80{ }^{\circ} \mathrm{C}$ and freeze-dried. The sample was gold-sputter coated for $30 \mathrm{~s}$ before being investigated under SEM (FEI USA).

(III) LSCM: FITC-conjugated GelMA was used to obtain fluorescent dye-labeled GelMA MSs. FITC-MSs and G5-AHP/CY5-miR-140 were colocalized using LSCM.

\section{Chondrocyte culture and tensile strain loading}

Chondrocytes were acquired from the knee articular cartilage of mice as previously reported ${ }^{48}$. Briefly, articular cartilage tissues were cut into small pieces $\left(<1 \mathrm{~mm}^{3}\right)$ and digested with $0.25 \%$ trypsin and $0.2 \%$ type II collagenase. The cells were obtained and cultured with DMEM/F12 media containing $10 \%$ fetal bovine serum (FBS) and antibiotics. Once the cells reached $80 \%$ confluence, they were subjected to cyclic tensile strain with a $0.5 \mathrm{~Hz}$ sinusoidal curve at $10 \%$ elongation for $24 \mathrm{~h}$ using a Flexcell1 FX-5000 ${ }^{\mathrm{Tm}}$ Tension System (Flexcell International Corporation, Burlington, NC).

\section{In vitro cell transfection}

Chondrocytes were seeded in 24-well plates and cultured for $24 \mathrm{~h}$ to reach $80 \%$ confluence. The G5-AHP/ miR-140 complexes mixed with $0.33 \mu \mathrm{g}$ CY5-miR-140 were diluted in $200 \mu \mathrm{l}$ of DMEM/F12 medium and incubated at room temperature for $10 \mathrm{~min}$. Afterwards, the cells were incubated with the complex solution for $6 \mathrm{~h}$ and then further cultured for $48 \mathrm{~h}$ with DMEM/F12 medium containing 10\% FBS and antibiotics. The expression of CY5- miR-140 in the cells was observed by LSCM (LSM800, Zeiss, Germany) and accurately analyzed by flow cytometry (BD FACSCalibur, San Jose). Cells transfected with Lipofectamine 3000 were used as positive controls.

\section{Quantification of mRNA and qRT-PCR}

Total RNA from chondrocytes was extracted using TRIzol reagent (Invitrogen, Carlsbad, California) as previously reported $^{48}$. One microgram of total RNA was used to synthesize cDNA using a RevertAid First Strand cDNA Synthesis Kit (TaKaRa, Dalian, China). The amplification of cDNA was performed by the SYBR Premix Ex Tag Kit (TaKaRa, Dalian, China). The primer sequences used in this study are shown in Supplementary Table S1. 


\section{Western blotting analysis}

Western blot analysis was accomplished as previously reported $^{49}$. Briefly, proteins were fractionated by SDSPAGE (7.5-12.5\% polyacrylamide gels) and transferred onto PVDF membranes (Millipore, Bedford, MA). The membranes were incubated with primary antibodies overnight at $4{ }^{\circ} \mathrm{C}$. The horseradish peroxidase-conjugated secondary antibody (1:5000) was used to react with the corresponding primary antibody for $1 \mathrm{~h}$. Antibody reactivity was observed using the Enhanced Chemiluminescence Western blot System (Amersham Biosciences). Image Processing and Analysis in Java (ImageJ) software was used to quantitatively evaluate the gray values of each band.

\section{Immunofluorescence staining}

The chondrocytes were fixed with $4 \%$ paraformaldehyde for $15 \mathrm{~min}$, followed by permeabilization with $0.1 \%$ Triton X100 for $15 \mathrm{~min}$. Subsequently, the cells were incubated with mouse antiCOL2 antibody (1:100 dilution), antiMMP13 antibody (1:100 dilution), and anti-ADAMTS5 antibody (1:100 dilution) at $4{ }^{\circ} \mathrm{C}$ overnight. The cells were washed with PBS three times and then incubated with appropriate Alexa Fluor-coupled secondary antibodies (Invitrogen, USA, 1:400) for $1 \mathrm{~h}$. The cell nuclei were stained with 4',6diamidino2phenylindole dilactate (Beyotime Biotechnology, China) at room temperature for 15 min. Finally, Alexa Fluor 594 phalloidin (Life Tech, USA) was used to label actin. The images were obtained by LSCM (LSM800, Zeiss, Germany).

\section{CCK-8 tests}

Chondrocytes were seeded in 12-well plates at a cell density of $4 \times 10^{4} \mathrm{ml}^{-1}$ and cocultured with G5-AHP/ miR-140 and MS@G5-AHP/miR-140 using Transwell chambers for 1,3 , and 5 days. All experiments were performed in triplicate. The Cell Counting Kit8 assay (Dojindo Laboratories, Kumamoto, Japan) was applied to investigate the cytotoxicity of MS@G5-AHP/miR-140 on chondrocytes. Briefly, $100 \mu \mathrm{l}$ of WST-1 cell proliferation reagent was added to each well followed by incubation for $2 \mathrm{~h}$ at $37^{\circ} \mathrm{C}$. Afterwards, the mixed medium was transferred to 96 -well plates. The optical density value of the samples was measured on a microplate reader (Infinite F50, Tecan, Switzerland). The absorbance value of the control group cells was defined as $100 \%$ survival.

\section{Live/dead staining}

The cell biocompatibility of MS@G5-AHP/miR-140 was measured by a Live/Dead Cell kit (Life Tech, USA). The cells were stained with $500 \mu \mathrm{l}$ of live/dead staining solution for $15 \mathrm{~min}$ and then observed using LSCM. Green fluorescence indicates viable cells with esterase activity, whereas red fluorescence shows dead cells. The live cell numbers were quantitatively analyzed using Image Processing and Analysis in Java (ImageJ) software.

\section{In vitro MMP-responsive tests}

(I) GelMA MSs were treated with or without $10 \mathrm{ng} / \mathrm{ml}$ MMPs, and the morphology of MSs at 1, 3, 5, and 7 days was assessed by BM.

(II) MS@G5-AHP/CY5-miR-140 was treated with or without $10 \mathrm{ng} / \mathrm{ml}$ MMPs. Briefly, $50 \mu \mathrm{l}$ of the solution was transferred to 96well plates away from light after treatment for 1, 3, 5, 7, and 14 days. The fluorescence intensity of each sample was measured on a microplate reader (Infinite F50, Tecan, Switzerland). The CY5-miR-140 contents proportional to fluorescence intensity were calculated according to the standard curve established between CY5-miR-140 content and G5-AHP/CY5-miR140 fluorescence intensity.

(III) Coculture of primary mouse chondrocytes with MS@G5-AHP/CY5-miR-140 using Transwell chambers was divided into two groups in triplicate. The Transwell + MMP group was treated with $10 \mathrm{ng} / \mathrm{ml}$ MMPs. The expression of CY5-miR-140 was visualized by LSCM after two days. The control group was treated only with G5AHP/miR-140.

\section{Animal experiments}

DMM surgery was applied to generate an animal model of OA in male C57 mice (8 weeks old; $n=25)^{50}$. After anesthetization, a medial articular approach was used to expose the arthrosis, and then, the quadriceps was gently turned laterally to avoid damaging the patellar ligament. Afterwards, the medial meniscus was dissociated, and the medial meniscus ligament (MMTL) was transected in a manner that did not injure the articular cartilage. Finally, the medial capsular incision was sutured after restoring the quadriceps well and closing the skin. Sham operations involving exposure of the joint capsule without MMTL transection were performed as a nonoperated control. The model mice were randomly divided into the PBS, MS@G5-AHP/miR-NC, G5-AHP/miR-140, and MS@G5AHP/miR-140 groups ( $n=5$ for each group) and intraarticularly injected once per fortnight with PBS, MS@G5-AHP/miR-NC, G5-AHP/miR-140, or MS@G5AHP/miR-140. All animal experiments were performed according to the protocol approved by the Animal Research Committee of Ruijin Hospital, School of Medicine, Shanghai Jiao Tong University, China, which complied with the National Institutes of Health Guidelines for the Care and Use of Laboratory Animals.

\section{Radiological evaluation and histological staining analyses}

Radiographical evaluation of mice with OA was conducted at 1 and 12 weeks after surgery, and digital plain radiographs of the morphology of mouse knees were 
acquired by an MX-20 Cabinet X-ray System (Faxitron, Tucson, AZ, USA). SkyScan1172 high-resolution microCT (Bruker, Kontich, Belgium) was applied to scan knee joints as previously described ${ }^{51}$. Reconstructed and threedimensional modeled data were obtained for further analysis. The samples were then dehydrated and embedded in paraffin. Six-micrometer sections were obtained by cutting tissues longitudinally. HE staining, toluidine blue staining and safranin Ofast green staining were used to analyze histological changes and proteoglycan content as reported previously ${ }^{52}$.

\section{Acknowledgements}

This work was supported by the National Key Research and Development Program of China (2020YFA0908200), the National Natural Science Foundation of China (grant numbers 81870617, 81930051, and 81803242), the Science and Technology Commission of Shanghai Municipality (20ZR1450600, 19440760400, and 18ZR1434200), the Research Project of Shanghai Municipal Health and Family Planning Commission (202040350), and GuangCi Professorship Program of Ruijin Hospital Shanghai Jiao Tong University School of Medicine.

\section{Author contributions}

B.L. mainly developed the concept, performed the experiment, wrote the rebuttal letter, and wrote the paper. F.W. analyzed the experimental results and wrote the paper. F.Q.H., T.D., P.H., X.X., J.L., C.W.L., Q.Z., M.L., and L.F.D. supported the experiment. L.G. and W.G.C. developed the concept, acquired the funding, and supervised the overall research.

\section{Conflict of interest}

The authors declare no competing interests.

\section{Publisher's note}

Springer Nature remains neutral with regard to jurisdictional claims in published maps and institutional affiliations.

Supplementary information The online version contains supplementary material available at https://doi.org/10.1038/s41427-021-00351-7.

Received: 20 April 2021 Revised: 2 December 2021 Accepted: 14 December 2021.

Published online: 7 January 2022

\section{References}

1. Kaufmann, K. B., Büning, H., Galy, A., Schambach, A. \& Grez, M. Gene therapy on the move. EMBO Mol. Med. 5, 1642-1661 (2013).

2. Ishikawa, K., Weber, T. \& Hajjar, R. J. Human cardiac gene therapy. Circulation Res. 123, 601-613 (2018).

3. O'Connor, D. M. \& Boulis, N. M. Gene therapy for neurodegenerative diseases. Trends Mol. Med. 21, 504-512 (2015).

4. Sudhakar, V. \& Richardson, R. M. Gene therapy for neurodegenerative diseases. Neurotherapeutics 16, 166-175 (2019).

5. Arruda, V. R. et al. Regional intravascular delivery of AAV-2-F.IX to skeletal muscle achieves long-term correction of hemophilia B in a large animal model. Blood 105, 3458-3464 (2005).

6. Emanueli, C. et al. Local delivery of human tissue kallikrein gene accelerates spontaneous angiogenesis in mouse model of hindlimb ischemia. Circulation 103, 125-132 (2001)

7. Sarett, S. M., Nelson, C. E. \& Duvall, C. L. Technologies for controlled, local delivery of siRNA. J. Control Release 218, 94-113 (2015).

8. Sano, A. et al. Atelocollagen for protein and gene delivery. Adv. Drug Deliv. Rev. 55, 1651-1677 (2003).

9. Cucchiarini, M. \& Madry, H. Biomaterial-guided delivery of gene vectors for targeted articular cartilage repair. Nat. Rev. Rheumatol. 15, 18-29 (2019).
10. Madry, H. et al. Thermosensitive hydrogel based on PEO-PPO-PEO poloxamers for a controlled in situ release of recombinant adeno-associated viral vectors for effective gene therapy of cartilage defects. Adv. Mater. 32, e1906508 (2020)

11. Jo, J. \& Tabata, Y. How controlled release technology can aid gene delivery. Expert Opin. Drug Deliv. 12, 1689-1701 (2015).

12. Yue, K. et al. Synthesis, properties, and biomedical applications of gelatin methacryloyl (GelMA) hydrogels. Biomaterials 73, 254-271 (2015).

13. Cheng, L. et al. Injectable polypeptide-protein hydrogels for promoting infected wound healing. Adv. Funct. Mater. 30, 2001196 (2020).

14. Yang, J. et al. Microfluidic liposomes-anchored microgels as extended delivery platform for treatment of osteoarthritis. Chem. Eng. J. 400, 126004 (2020)

15. De Geest, B. G., Urbanski, J. P., Thorsen, T., Demeester, J. \& De Smedt, S. C. Synthesis of monodisperse biodegradable microgels in microfluidic devices. Langmuir 21, 10275-10279 (2005).

16. Daly, A. C., Riley, L., Segura, T. \& Burdick, J. A. Hydrogel microparticles for biomedical applications. Nat. Rev. Mater. 5, 20-43 (2020).

17. Shang, L., Cheng, Y. \& Zhao, Y. Emerging droplet microfluidics. Chem. Rev. 117, 7964-8040 (2017)

18. Jalalvandi, E. \& Shavandi, A. Shear thinning/self-healing hydrogel based on natural polymers with secondary photocrosslinking for biomedical applications. J. Mech. Behav. Biomed. Mater. 90, 191-201 (2019).

19. Chen, D. et al. Osteoarthritis: toward a comprehensive understanding of pathological mechanism. Bone Res. 5, 16044 (2017).

20. Loeser, R. F, Goldring, S. R. Scanzello, C. R. \& Goldring, M. B. Osteoarthritis: a disease of the joint as an organ. Arthritis Rheum. 64, 1697-1707 (2012).

21. Kim, J. H. et al. Regulation of the catabolic cascade in osteoarthritis by the zincZIP8-MTF1 axis. Cell 156, 730-743 (2014).

22. Nugent, M. MicroRNAs: exploring new horizons in osteoarthritis. Osteoarthr. Cartil. 24, 573-580 (2016).

23. $\mathrm{Yu}, \mathrm{C} ., \mathrm{Chen}, \mathrm{W}$. P. \& Wang, X. H. MicroRNA in osteoarthritis. J. Int. Med. Res. 39 1-9 (2011).

24. Miyaki, S. et al. MicroRNA-140 plays dual roles in both cartilage development and homeostasis. Genes Dev. 24, 1173-1185 (2010).

25. Zhang, R., Ma, J. \& Yao, J. Molecular mechanisms of the cartilage-specific microRNA-140 in osteoarthritis. Inflamm. Res. 62, 871-877 (2013).

26. Papaioannou, $\mathrm{G}$. et al. MicroRNA-140 provides robustness to the regulation of hypertrophic chondrocyte differentiation by the PTHrP-HDAC4 pathway. J. Bone Miner. Res. 30, 1044-1052 (2015).

27. Madry, H. \& Cucchiarini, M. Gene therapy for human osteoarthritis: principles and clinical translation. Expert Opin. Biol. Ther. 16, 331-346 (2016).

28. Evans, C. H., Gouze, J. N., Gouze, E., Robbins, P. D. \& Ghivizzani, S. C. Osteoarthritis gene therapy. Gene Ther. 11, 379-389 (2004).

29. Gupta, V., Khan, Y., Berkland, C. J., Laurencin, C. T. \& Detamore, M. S. Microsphere-based scaffolds in regenerative engineering. Annu Rev. Biomed. Eng. 19, 135-161 (2017).

30. Ribeiro, J. S. et al. Injectable MMP-responsive nanotube-modified gelatin hydrogel for dental infection ablation. ACS Appl Mater. Interfaces 12, 16006-16017 (2020).

31. Zhao, $X$. et al. Photoresponsive delivery microcarriers for tissue defects repair. Adv. Sci. Weinh., Baden.-Wurtt., Ger.) 6, 1901280 (2019).

32. Qiao, Y. et al. Gelatin templated polypeptide co-cross-linked hydrogel for bone regeneration. Adv. Healthc. Mater. 9, e1901239 (2020).

33. Rupaimoole, R. \& Slack, F. J. MicroRNA therapeutics: towards a new era for the management of cancer and other diseases. Nat. Rev. Drug Discov. 16, 203-222 (2017).

34. Chang, H., Zhang, J., Wang, H., LV, J. \& Cheng, Y. A combination of guanidyl and phenyl groups on a dendrimer enables efficient siRNA and DNA delivery. Biomacromolecules 18, 2371-2378 (2017)

35. Wang, F., Deng, L., Hu, J. \& Cheng, Y. Being two is better than being one: a facile strategy to fabricate multicomponent nanoparticles for efficient gene delivery. Bioconjug. Chem. 27, 638-646 (2016).

36. Wang, F., Hu, K. \& Cheng, Y. Structure-activity relationship of dendrimers engineered with twenty common amino acids in gene delivery. Acta Biomater. 29, 94-102 (2016).

37. Wang, F. et al. Synergistic effect of amino acids modified on dendrimer surface in gene delivery. Biomaterials 35, 9187-9198 (2014).

38. Yu, G. S. et al. Synthesis of PAMAM dendrimer derivatives with enhanced buffering capacity and remarkable gene transfection efficiency. Bioconjug. Chem. 22, 1046-1055 (2011). 
39. Chang, $H$. et al. Rational design of a polymer with robust efficacy for intracellular protein and peptide delivery. Nano Lett. 17, 1678-1684 (2017).

40. Dufès, C., Uchegbu, I. F. \& Schätzlein, A. G. Dendrimers in gene delivery. Adv. Drug Deliv. Rev. 57, 2177-2202 (2005).

41. Carballo, C. B., Nakagawa, Y., Sekiya, I. \& Rodeo, S. A. Basic science of articular cartilage. Clin. Sports Med. 36, 413-425 (2017)

42. Chen, K. et al. Increased 15-lipoxygenase-1 expression in chondrocytes contributes to the pathogenesis of osteoarthritis. Cell Death Dis. 8, e3109 (2017).

43. Gao, Y., Ji, H., Peng, L., Gao, X., Jiang, S. Development of PLGA-PEG-PLGA hydrogel delivery system for enhanced immunoreaction and efficacy of newcastle disease virus DNA vaccine. Molecules (Basel, Switzerland). 25, 2505 (2020).

44. Ning, P. et al. High encapsulation and localized delivery of curcumin from an injectable hydrogel. Mater. Sci. Eng. C., Mater. Biol. Appl. 83, 121-129 (2018).

45. Puckert, C. et al. Electro-mechano responsive properties of gelatin methacrylate (GelMA) hydrogel on conducting polymer electrodes quantified using atomic force microscopy. Soft Matter 13, 4761-4772 (2017).
46. Bravo-Osuna, I. et al. Microspheres as intraocular therapeutic tools in chronic diseases of the optic nerve and retina. Adv. Drug Deliv. Rev. 126, 127-144 (2018).

47. Wei, D. X., Dao, J. W. \& Chen, G. Q. A micro-Ark for cells: highly open porous polyhydroxyalkanoate microspheres as injectable scaffolds for tissue regeneration. Adv. Mater. 30, e1802273 (2018).

48. Song, T. et al. Regulation of chondrocyte functions by transient receptor potential cation channel V6 in osteoarthritis. J. Cell Physiol. 232, 3170-3181 (2017).

49. Cho, H., Gallaher, D. D. \& Csallany, A. S. Conjugated linoleic acid, cis-9,trans-11, is a substrate for pulmonary 15-lipoxygenase-1 in rat. J. Agric. Food Chem. $\mathbf{5 3}$ 7262-7266 (2005)

50. Lorenz, J. \& Grässel, S. Experimental osteoarthritis models in mice. Methods Mol. Biol. 1194, 401-419 (2014).

51. Cui, Z. et al. Halofuginone attenuates osteoarthritis by inhibition of TGF- $\beta$ activity and $\mathrm{H}$-type vessel formation in subchondral bone. Ann. Rheum. Dis. 75, 1714-1721 (2016)

52. Pritzker, K. P. et al. Osteoarthritis cartilage histopathology: grading and staging. Osteoarthr. Cartil. 14, 13-29 (2006). 